International Journal of Pure and Applied Mathematics

Volume 92 No. 2 2014, 191-206

ISSN: 1311-8080 (printed version); ISSN: 1314-3395 (on-line version)

url: http://www.ijpam.eu

doi: http://dx.doi.org/10.12732/ijpam.v92i2.5

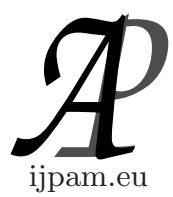

\title{
THE LENGTH-BIASED EXPONENTIATED INVERTED WEIBULL DISTRIBUTION
}

\author{
Palakorn Seenoi ${ }^{1}$, Thidaporn Supapakorn ${ }^{2}$, Winai Bodhisuwan ${ }^{3} \S$ \\ $1,2,3$ Department of Statistics \\ Kasetsart University \\ Chatuchak, Bangkok, 10900, THAILAND
}

\begin{abstract}
This paper develops a length-biased of the exponentiated inverted Weibull (EIW) distribution, it is named as the length-biased exponentiated inverted Weibull (LBEIW). Some probability functions are derived, including the moments of this distribution. The length-biased inverted Weibull (LBIW) distribution is presented as a special case of the LBEIW distribution. Maximum likelihood estimation method is employed to parameter estimation. Finally, a real dataset of distance between cracks in a pipe is used to illustrate by fitting the LBEIW, EIW, LBIW and Weibull distributions to the data. It is shown that the LBEIW and EIW distributions can be fitted well.
\end{abstract}

AMS Subject Classification: 60E05

Key Words: length-biased, exponentiated inverted weibull distribution, hazard rate, maximum likelihood estimation, goodness of fit test

\section{Introduction}

The Weibull distribution was introduced by Wallodi Weibull, Swedish scientist, in 1951 [1]. It is perhaps the most widely used distribution to analyze the

Received: November 26, 2013

(C) 2014 Academic Publications, Ltd.

$\S$ Correspondence author url: www.acadpubl.eu 
lifetime data. This distribution provides vast impact of reliability and quality control, such as ball bearings, automobile components, concrete bridges, demography, actuarial study, and electrical insulation. It is also used in biological and medical applications, for example, in the studies of occurrence of tumors in human populations and in laboratory animals. Hence, applications to the lifetime or durability of manufactured items are common.

Let $T$ be a random variable with Weibull distribution. Its probability density function (pdf) takes the form:

$$
f(t)=\lambda \beta(\lambda t)^{\beta-1} \exp \left\{-(\lambda t)^{\beta}\right\} ; \quad t>0,
$$

where $\lambda>0$ and $\beta>0$ are scale and shape parameters, respectively. This distribution includes especially the exponential and the Rayleigh distributions as special cases where $\beta=1$ and $\beta=2$, respectively. The Weibull distribution has a distribution function of the form:

$$
F(t)=1-\exp \left\{-(\lambda t)^{\beta}\right\} .
$$

The survival function and hazard rate are, respectively,

$$
S(t)=\exp \left\{-(\lambda t)^{\beta}\right\}
$$

and

$$
h(t)=\lambda \beta(\lambda t)^{\beta-1} .
$$

The hazard rate has several forms, e.g., decreasing, constant, increasing, bathtub shape, $\mathrm{W}$ shape, etc. The Weibull hazard rate is monotone increasing if $\beta>1$, monotone decreasing if $\beta<1$, and constant when $\beta=1$. The Weibull distribution is fairly flexible in providing a good description of many types of lifetime data [2] such as complete data, censored data. Censored data include right, left, or interval censoring, type I or type II censoring, and single or multiple censoring [3].

In some cases the Weibull distribution may not be fit to some datasets, however, alternatively, the inverse Gaussian, gamma, inverse gamma, log-normal distributions can be good representative. In addition, many studies proposed various lifetime distributions. Keller and Kamath studied the shapes of the density and hazard rate for the basic inverse model in 1982 [4]. Also, the inverted Weibull (IW) distribution was proposed and applied to a wide range of situations including applications in medicine, reliability and ecology. Many researchers applied IW distribution to their work, e.g., Erto [5] has shown that 
the IW distribution gave a good fit to life testing data reported by Nelson. Furthermore, Khan et al. [6] explained the flexibility of the three parameters inverted Weibull distribution and its interested properties.

An exponentiated distribution is a generalization of the distribution through adding a new shape parameter $\theta \in \Re^{+}$, i.e., $\theta>0$, by exponentiating the distribution function $F$ in the form $F^{\theta}$. The exponentiated Weibull distribution was proposed by Mudholkar and Srivastava [7] as an extension of the Weibull family obtained by adding the second shape parameter. Mudholkar et al. [8] applied the exponentiated Weibull distribution to serve survival data and showed that the hazard rate are increasing, decreasing, bathtub shape, and unimodal. The exponentiated exponential distribution proposed by Gupta and Kundu [9] is a special case of the exponentiated Weibull family. Flaih et al. [10] extended the IW distribution to the exponentiated inverted Weibull (EIW) distribution by adding another shape parameter. This study suggested that the EIW distribution can provide a better fit to the real dataset than the IW distribution.

In this paper, the length-biased exponentiated inverted Weibull (LBEIW) distribution is proposed. The rest of the paper is organized as follows: in Section 2, the EIW distribution is introduced. The LBEIW is defined in Section 3. The $k^{\text {th }}$ moment of this distribution is investigated in Section 4. In Section 5, we provide some special cases of the LBEIW distribution. In Section 6, we propose algorithm to generate random data of the LBEIW distribution. In Section 7, we discuss maximum likelihood estimation (MLE) to estimate parameters of the LBEIW distribution. Finally, a goodness of fit test of the LBEIW distribution and its application are provided in Section 8.

\section{Exponentiated Inverted Weibull Distribution}

The EIW distribution was proposed by Flaih et al. [10]. The EIW generalizes the IW distribution and it has two shape parameters. If $X$ has EIW distribution, its distribution function takes the following form:

$$
F(x)=\left\{\exp \left(-x^{-\beta}\right)\right\}^{\theta} ; \quad x>0, \beta>0, \theta>0 .
$$

The pdf of $X$ is:

$$
f(x)=\theta \beta x^{-(\beta+1)}\left\{\exp \left(-x^{-\beta}\right)\right\}^{\theta} .
$$

The hazard rate of the EIW distribution is given by:

$$
h(x)=\frac{\theta \beta x^{-(\beta+1)}\left\{\exp \left(-x^{-\beta}\right)\right\}^{\theta}}{1-\left\{\exp \left(-x^{-\beta}\right)\right\}^{\theta}} .
$$


The $k^{\text {th }}$ moment of the EIW distribution is given as the following:

$$
E_{f}\left(X^{k}\right)=\theta^{\frac{k}{\beta}} \Gamma\left(1-\frac{k}{\beta}\right) ; \beta>k,
$$

where $\Gamma(z)=\int_{0}^{\infty} t^{z-1} \exp (-t) \mathrm{d} t$ is a gamma function.

Then, the expected value of $X$ can be written as:

$$
E_{f}(X)=\theta^{\frac{1}{\beta}} \Gamma\left(1-\frac{1}{\beta}\right) ; \beta>1 .
$$

For $\theta=1$, the EIW distribution represents the IW distribution, when $\beta=1$ it represents the exponentiated inverted exponential distribution. Thus, the EIW distribution is a generalization of the exponentiated inverted exponential distribution as well as the IW distribution.

\section{Length-Biased Exponentiated Inverted Weibull Distribution}

The concept of length-biased was introduced by Cox in 1962 [11]. This concept is found in various applications in biomedical area such as family history and disease, survival analysis, intermediate events and latency period of AIDS due to blood transfusion [12]. Many works were done to characterize relationships between original distributions and their length-biased versions. Patill and Rao expressed some basic distributions and their length-biased forms such as log-normal, gamma, pareto, beta distributions [13]. Recently, many researches are applied to length-biased for lifetime distribution, length-biased weighted Weibull distribution [14], length-biased weighted generalized Rayleigh distribution [15], length-biased beta distribution [16], and Bayes estimation of lengthbiased Weibull distribution [17].

In this study, we propose a new distribution which is a LBEIW distribution. We first provide a general definition of the LBEIW distribution which will subsequently reveal its pdf.

Definition 1. If $X$ has a lifetime distribution with pdf $f(x)$ and expected value, $E_{f}(X)<\infty$, the pdf of length-biased distribution of $X$ can be defined as:

$$
g(x)=\frac{x f(x)}{E_{f}(X)} .
$$

Theorem 2. Let $X$ be a random variable of an EIW distribution with pdf $f(x)$. Then $g(x)=\frac{x f(x)}{E_{f}(X)}$ is a pdf of the LBEIW distribution with two 
shape parameters $\beta$ and $\theta$. The notation for $X$ with the LBEIW distribution is denoted as $X \sim \operatorname{LBEIW}(\beta, \theta)$. The $p d f$ of $X$ is given by:

$$
g(x)=\frac{\beta \theta^{1-\frac{1}{\beta}}}{\Gamma\left(1-\frac{1}{\beta}\right)} x^{-\beta}\left\{\exp \left(-x^{-\beta}\right)\right\}^{\theta} ; x>0, \beta>1, \theta>0 .
$$

Proof. By definition 1, substitute (2) and (5) into (6), then the pdf for the LBEIW distribution can be obtained by:

$$
\begin{aligned}
g(x) & =\frac{x}{\theta^{\frac{1}{\beta}} \Gamma\left(1-\frac{1}{\beta}\right)} \theta \beta x^{-(\beta+1)}\left\{\exp \left(-x^{-\beta}\right)\right\}^{\theta} \\
& =\frac{\beta \theta^{1-\frac{1}{\beta}}}{\Gamma\left(1-\frac{1}{\beta}\right)} x^{-\beta}\left\{\exp \left(-x^{-\beta}\right)\right\}^{\theta} .
\end{aligned}
$$

The pdf plots of the LBEIW distribution are shown in Figure 1 which can be seen as a unimodal and positively skewed.

Theorem 3. Let $X$ be a random variable of the $L B E I W$ distribution with parameters $\beta$ and $\theta$. The distribution function of the LBEIW distribution is written as:

$$
G(x)=\frac{\Gamma\left(1-\frac{1}{\beta}, \frac{\theta}{x^{\beta}}\right)}{\Gamma\left(1-\frac{1}{\beta}\right)},
$$

where $\Gamma(s, x)=\int_{x}^{\infty} t^{s-1} e^{-t} \mathrm{~d} t$ is an upper incomplete gamma function.

Proof. Generally, the distribution function of lifetime distribution is defended as:

$$
G(x)=\int_{0}^{x} g(t) d t
$$

Substituting (7) into (9), we obtain:

$$
G(x)=\int_{0}^{x} \frac{\beta \theta^{1-\frac{1}{\beta}}}{\Gamma\left(1-\frac{1}{\beta}\right)} t^{-\beta}\left\{\exp \left(-t^{-\beta}\right)\right\}^{\theta} d t .
$$



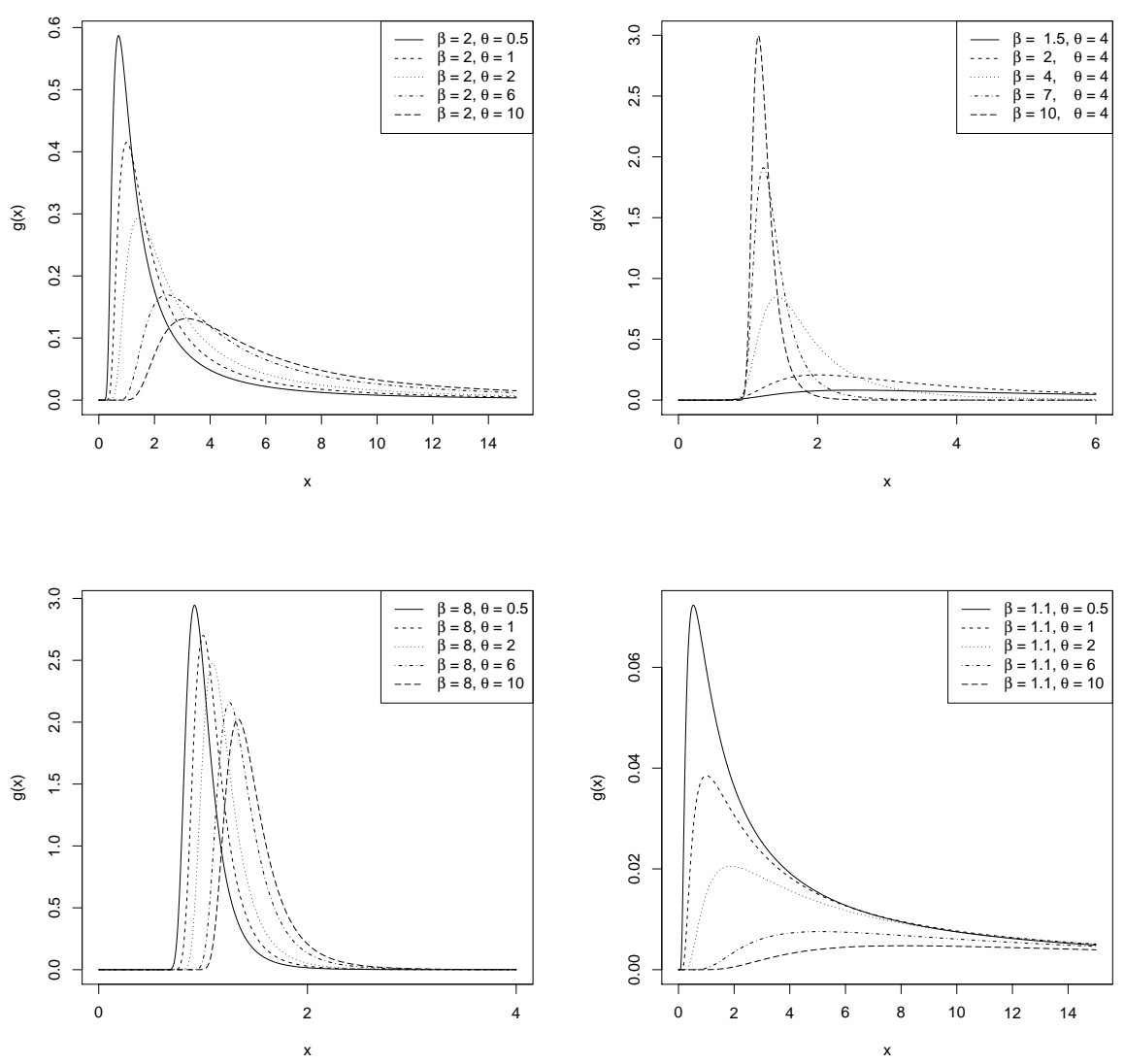

Figure 1: The probability density function of the LBEIW distribution for selected values of $\beta$ and $\theta$.

By setting $u=\theta t^{-\beta}, \frac{\theta}{x^{\beta}}<u<\infty$, the above integration becomes:

$$
\begin{aligned}
G(x) & =\frac{1}{\Gamma\left(1-\frac{1}{\beta}\right) \theta^{\frac{1}{\beta}}} \int_{\frac{\theta}{x^{\beta}}}^{\infty} \frac{\theta^{\frac{1}{\beta}}}{u^{\frac{1}{\beta}}} \exp (-u) d u \\
& =\frac{1}{\Gamma\left(1-\frac{1}{\beta}\right)} \int_{\frac{\theta}{x^{\beta}}}^{\infty} u^{-\frac{1}{\beta}} \exp (-u) d u
\end{aligned}
$$




$$
\begin{aligned}
& =\frac{1}{\Gamma\left(1-\frac{1}{\beta}\right)} \int_{\frac{\theta}{x^{\beta}}}^{\infty} u^{\left(1-\frac{1}{\beta}\right)-1} \exp (-u) d u \\
& =\frac{\Gamma\left(1-\frac{1}{\beta}, \frac{\theta}{x^{\beta}}\right)}{\Gamma\left(1-\frac{1}{\beta}\right)} .
\end{aligned}
$$

Some plots of the LBEIW distribution function with specific parameter values are shown in Figure 2.

Theorem 4. Let $X$ be a random variable of the $L B E I W$ distribution with parameters $\beta$ and $\theta$. The survival function of the LBEIW distribution can be written as:

$$
S(x)=\frac{\gamma\left(1-\frac{1}{\beta}, \frac{\theta}{x^{\beta}}\right)}{\Gamma\left(1-\frac{1}{\beta}\right)},
$$

where $\gamma(s, x)=\int_{0}^{x} t^{s-1} \exp (-t) \mathrm{d} t$ is a lower incomplete gamma function.

Proof. By definition, the survival function of the random variable $X$ is given by:

$$
S(x)=1-G(x) .
$$

Using (8), the survival function of the LBEIW distribution can be expressed by:

$$
\begin{aligned}
S(x) & =1-\frac{\Gamma\left(1-\frac{1}{\beta}, \frac{\theta}{x^{\beta}}\right)}{\Gamma\left(1-\frac{1}{\beta}\right)} \\
& =\frac{\Gamma\left(1-\frac{1}{\beta}\right)-\Gamma\left(1-\frac{1}{\beta}, \frac{\theta}{x^{\beta}}\right)}{\Gamma\left(1-\frac{1}{\beta}\right)} \\
& =\frac{\gamma\left(1-\frac{1}{\beta}, \frac{\theta}{x^{\beta}}\right)}{\Gamma\left(1-\frac{1}{\beta}\right)} .
\end{aligned}
$$



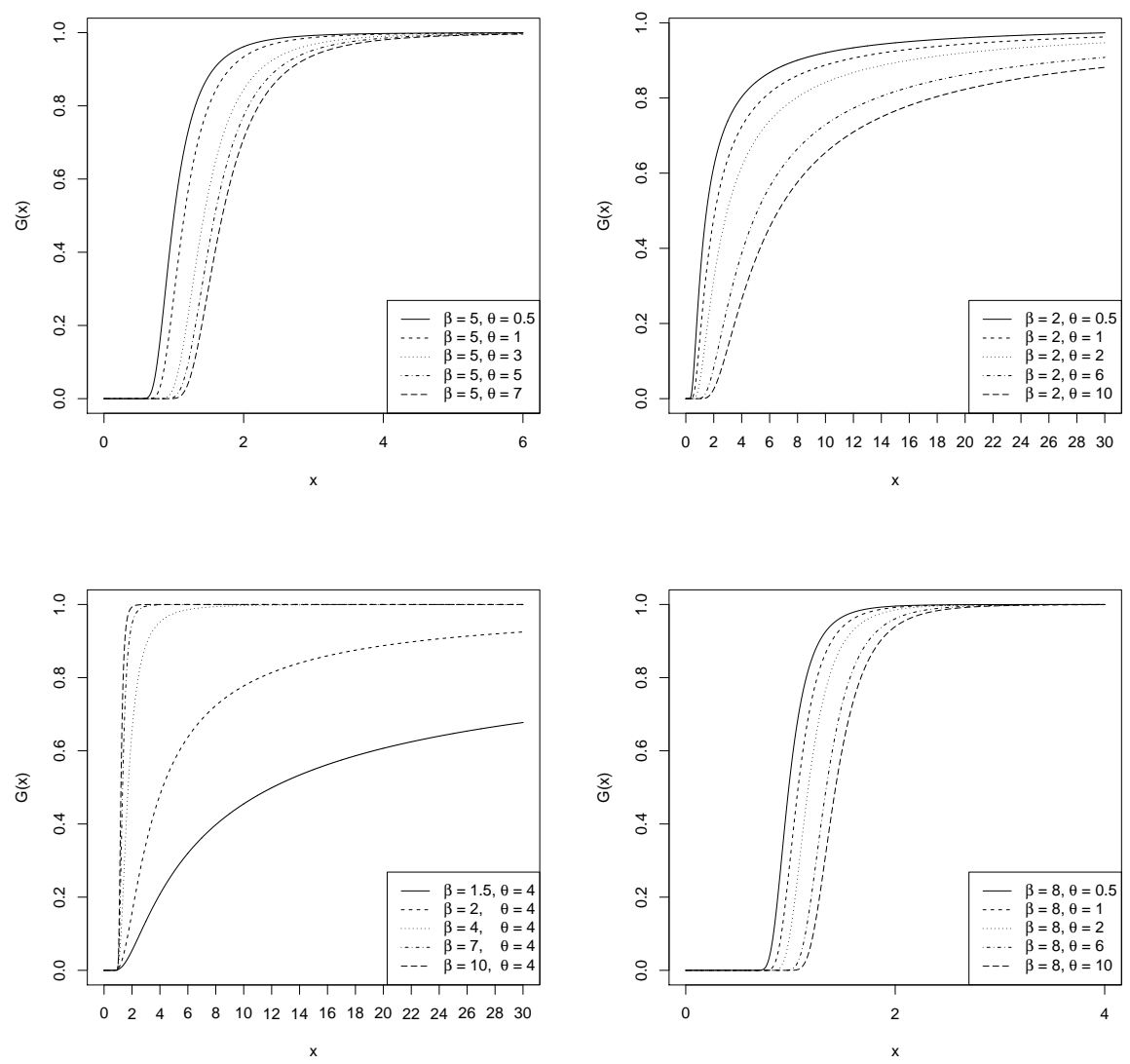

Figure 2: The distribution function of the LBEIW distribution for selected values of $\beta$ and $\theta$.

Theorem 5. Let $X$ be a random variable of the $L B E I W$ distribution with parameters $\beta$ and $\theta$. The hazard rate of the $L B E I W$ distribution takes the form:

$$
h(x)=\frac{\beta \theta^{1-\frac{1}{\beta}} x^{-\beta}\left\{\exp \left(-x^{-\beta}\right)\right\}^{\theta}}{\gamma\left(1-\frac{1}{\beta}, \frac{\theta}{t^{\beta}}\right)} .
$$

Proof. Let $X$ be a continuous random variable with pdf and survival function, $g(x)$ and $S(x)$, respectively, then the hazard rate is defined by:

$$
h(x)=\frac{g(x)}{S(x)} .
$$


Substituting (7) and (10) into (12), we obtain:

$$
\begin{aligned}
h(x) & =\frac{\beta \theta^{1-\frac{1}{\beta}} x^{-\beta}\left\{\exp \left(-x^{-\beta}\right)\right\}^{\theta} / \Gamma\left(1-\frac{1}{\beta}\right)}{\gamma\left(1-\frac{1}{\beta}, \frac{\theta}{x^{\beta}}\right) / \Gamma\left(1-\frac{1}{\beta}\right)} \\
& =\frac{\beta \theta^{1-\frac{1}{\beta}} x^{-\beta}\left\{\exp \left(-x^{-\beta}\right)\right\}^{\theta}}{\gamma\left(1-\frac{1}{\beta}, \frac{\theta}{x^{\beta}}\right)} .
\end{aligned}
$$

Some hazard rate plots of the LBEIW distribution with specific parameter values are displayed in Figure 3.

\section{The $k^{\text {th }}$ Moment of the LBEIW Distribution}

The result of this section gives the $k^{\text {th }}$ moment of LBEIW distribution.

Theorem 6. If $X \sim \operatorname{LBEIW}(\beta, \theta)$, then the $k^{\text {th }}$ moment of $X$ is given as follow:

$$
E_{g}\left(X^{k}\right)=\theta^{\frac{k}{\beta}} \frac{\Gamma\left(1-\frac{k+1}{\beta}\right)}{\Gamma\left(1-\frac{1}{\beta}\right)}, \quad k=1,2,3, \ldots
$$

Proof. Let $X$ is an absolutely continuous non-negative random variable with pdf $g(x)$, then $k^{\text {th }}$ moment of $X$ can be obtained by:

$$
E_{g}\left(X^{k}\right)=\int_{0}^{\infty} x^{k} g(x) d x .
$$

From the pdf of the LBEIW distribution in (7), then shows that $E_{g}\left(X^{k}\right)$ can be written as:

$$
\begin{aligned}
E_{g}\left(X^{k}\right) & =\int_{0}^{\infty} x^{k} \frac{\beta \theta^{1-\frac{1}{\beta}}}{\Gamma\left(1-\frac{1}{\beta}\right)} x^{-\beta}\left\{\exp \left(-x^{-\beta}\right)\right\}^{\theta} d x \\
& =\frac{(-1)}{\Gamma\left(1-\frac{1}{\beta}\right) \theta^{\frac{1}{\beta}}} \int_{\infty}^{0} \frac{\theta^{\frac{k+1}{\beta}}}{u^{\frac{k+1}{\beta}}} \exp (-u) d u \\
& =\frac{\theta^{\frac{k}{\beta}}}{\Gamma\left(1-\frac{1}{\beta}\right)} \int_{0}^{\infty} u^{-\frac{k+1}{\beta}} \exp (-u) d u
\end{aligned}
$$



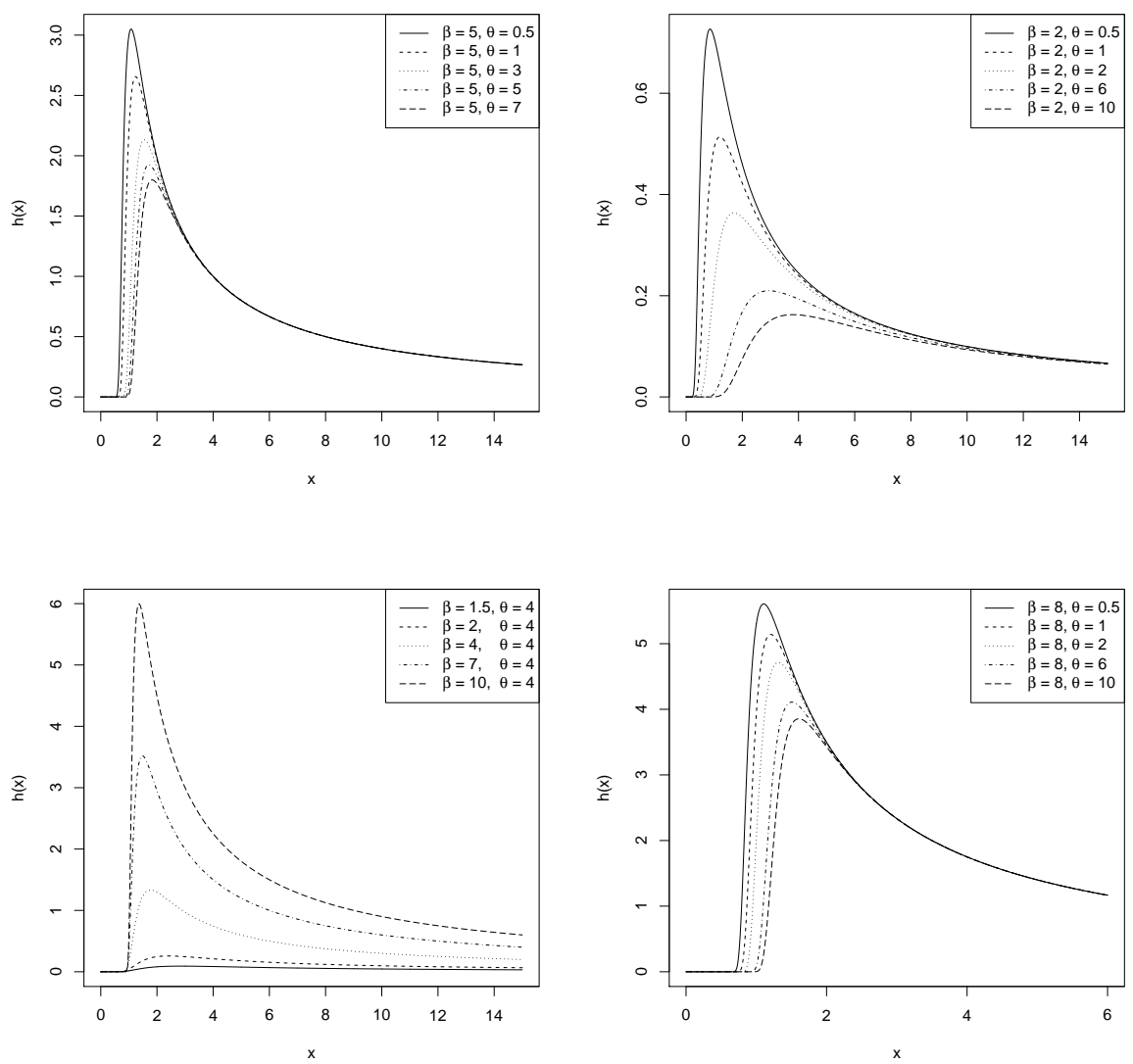

Figure 3: The hazard rate of the LBEIW distribution for selected values of $\beta$ and $\theta$.

$$
\begin{aligned}
& =\frac{\theta^{\frac{k}{\beta}}}{\Gamma\left(1-\frac{1}{\beta}\right)} \int_{0}^{\infty} u^{\left(1-\frac{k+1}{\beta}\right)-1} \exp (-u) d u \\
& =\theta^{\frac{k}{\beta}} \frac{\Gamma\left(1-\frac{k+1}{\beta}\right)}{\Gamma\left(1-\frac{1}{\beta}\right)} .
\end{aligned}
$$

From the $k^{\text {th }}$ moment of the LBEIW distribution, putting $k=1$ in (13), the expected value of $X$ is obtained as: 


$$
E_{g}(X)=\theta^{\frac{1}{\beta}} \frac{\Gamma\left(1-\frac{2}{\beta}\right)}{\Gamma\left(1-\frac{1}{\beta}\right)} .
$$

\section{A Special Case of the LBEIW Distribution}

If $X$ is a random variable with LBEIW distribution, the special case of $X$ can be seen as corollary below.

Corollary 7. For $\theta=1$, we get the length-biased inverted Weibull (LBIW) distribution with pdf given by:

$$
g(x)=\frac{\beta}{\Gamma\left(1-\frac{1}{\beta}\right)} x^{-\beta}\left\{\exp \left(-x^{-\beta}\right)\right\} .
$$

Proof. Substituting $\theta=1$ in (7), we get:

$$
\begin{aligned}
g(x) & =\frac{\beta 1^{1-\frac{1}{\beta}}}{\Gamma\left(1-\frac{1}{\beta}\right)} x^{-\beta}\left\{\exp \left(-x^{-\beta}\right)\right\}^{1} \\
& =\frac{\beta}{\Gamma\left(1-\frac{1}{\beta}\right)} x^{-\beta}\left\{\exp \left(-x^{-\beta}\right)\right\} .
\end{aligned}
$$

\section{Random Variate Generation}

We use inverse transformation technique to generate random data from LBEIW distribution by setting:

$$
U=G^{-1}(x),
$$

where $U$ is distributed as uniform distribution on $(0,1)$, denoted as $U(0,1)$.

The distribution function of the LBEIW distribution can be written in term of:

$$
G(x)=\frac{\Gamma\left(1-\frac{1}{\beta}, \frac{\theta}{x^{\beta}}\right)}{\Gamma\left(1-\frac{1}{\beta}\right)} .
$$

To generate random data $X_{i}, i=1, \ldots, n$, from $\operatorname{LBEIW}(\beta, \theta)$, one can use the following algorithm: 
1. Generate $U_{i}, i=1, \ldots, n$, from $U(0,1)$.

2. Set $U_{i}=G\left(x_{i}\right)$, then $U_{i}=\frac{\Gamma\left(1-\frac{1}{\beta}, \frac{\theta}{x_{i}^{\beta}}\right)}{\Gamma\left(1-\frac{1}{\beta}\right)}$ and $U_{i} \Gamma\left(1-\frac{1}{\beta}\right)=\Gamma\left(1-\frac{1}{\beta}, \frac{\theta}{x_{i} \beta}\right)$.

3. Assigned $v_{i}=U_{i} \Gamma\left(1-\frac{1}{\beta}\right)$.

4. Set $a_{i}=\operatorname{Igamma.Inv}\left(1-\frac{1}{\beta}, v_{i}\right)$, when Igamma.Inv is inverted of incomplete gamma function in $\mathrm{R}$ program.

5. Set $a_{i}=\frac{\theta}{x_{i}{ }^{\beta}}$.

6. Then, $X_{i}=\left(\frac{\theta}{a_{i}}\right)^{\frac{1}{\beta}}$.

\section{Parameter Estimation}

In this section, we derive the non-linear equations for finding the maximum likelihood estimates of the parameters. It is assumed that $X$ follows $\operatorname{LBEIW}(\beta, \theta)$ and $(\beta, \theta)^{T}$ denotes the parameter vector. The estimation of parameters for the LBEIW distribution is done via the MLE. We will provide the MLE procedure as follows:

The likelihood function of the $\operatorname{LBEIW}(\beta, \theta)$ is given by:

$$
L(\beta, \theta ; x)=\frac{\beta^{n} \theta^{n\left(1-\frac{1}{\beta}\right)}}{\left[\Gamma\left(1-\frac{1}{\beta}\right)\right]^{n}} \prod_{i=1}^{n} x_{i}^{-\beta} \exp \left(-\theta \sum_{i=1}^{n} x_{i}^{-\beta}\right) .
$$

The log-likelihood function of $n$ observations of $X$ can be written as:

$$
\begin{aligned}
l(\beta, \theta ; x)= & \log L(\beta, \theta ; x) \\
= & n \log \beta+n \log \theta-\frac{n}{\beta} \log \theta-\beta \sum_{i=1}^{n} \log x_{i} \\
& -\theta \sum_{i=1}^{n} x_{i}^{-\beta}-n \log \Gamma\left(1-\frac{1}{\beta}\right) .
\end{aligned}
$$

Let $\psi(z)=\frac{d}{d z} \log \Gamma(z)=\frac{\Gamma^{\prime}(z)}{\Gamma(z)}$ be a digamma function, which is the logarithmic derivative of the gamma function. Then the components of the unit score vector $U_{n}=((\partial l / \partial \beta),(\partial l / \partial \theta))^{T}$ are given by: 


$$
\begin{aligned}
\frac{\partial l}{\partial \beta}= & \frac{n}{\beta}+\frac{n}{\beta^{2}} \log \theta-\sum_{i=1}^{n} \log x_{i} \\
& +\theta\left[\sum_{i=1}^{n} x_{i}^{-\beta}\right]\left[\sum_{i=1}^{n} \log x_{i}\right]-n \psi\left(1-\frac{1}{\beta}\right), \\
\frac{\partial l}{\partial \theta}= & \frac{n}{\theta}-\frac{n}{\beta \theta}-\sum_{i=1}^{n} x_{i}^{-\beta}
\end{aligned}
$$

The MLE solutions of $(\beta, \theta)^{T}$ can be obtained numerically from the nonlinear equations by setting $U_{n}=0$ and solving the equations (18) and (19) simultaneously using a numerical procedure with the Newton-Raphson method.

\section{Data Analysis}

This section presents the comparison analysis of the LBEIW, EIW, LBIW and Weibull distributions applying to the real datasets. We consider uncensored data on distance between cracks in a pipe dataset [2]. The data is given in Table 1. We can also perform formal goodness of fit test in order to verify which distribution fits to the data. We apply the Kolmogorov-Smirnov test (KS test) [18] and Anderson-Darling test (AD test) [19] for the goodness of fit purpose. Table 2 lists the MLE estimates of the parameters $\beta$ and $\theta$ and values of the test statistics which are KS test and AD test. The p-values of KS and $\mathrm{AD}$ tests for the LBEIW distribution are 0.9376 and 0.9447 , respectively. The results in Table 2 shown that the LBEIW distribution fits the data as well as the EIW distribution.

Table 1: Distance between cracks in a pipe dataset.

\begin{tabular}{llllllll}
\hline \hline 30.94 & 18.51 & 16.62 & 51.56 & 22.85 & 22.38 & 19.08 & 49.56 \\
17.12 & 10.67 & 25.43 & 10.24 & 27.47 & 14.70 & 14.10 & 29.93 \\
27.98 & 36.02 & 19.40 & 14.97 & 22.57 & 12.26 & 18.14 & 18.84 \\
\hline \hline
\end{tabular}

In addition, the probability plot of the LBEIW distribution, corresponding to the distance between cracks in a pipe dataset, is shown in the Figure 4. 
Table 2: Goodness of fit summary of distance between cracks in a pipe dataset.

\begin{tabular}{crrrrrrr}
\hline \hline Fitting & \multicolumn{2}{c}{ KS test } & \multicolumn{2}{c}{ AD test } & \multicolumn{3}{c}{ Estimate } \\
\cline { 2 - 7 } Distribution & Statistic & p-value & Statistic & p-value & $\beta$ & $\theta$ & $\lambda$ \\
\hline LBEIW & 0.1031 & 0.9376 & 0.2905 & 0.9447 & 3.3891 & 9508.9505 & - \\
EIW & 0.0891 & 0.9822 & 0.2213 & 0.9835 & 2.7347 & 2384.5601 & - \\
LBIW & 0.5129 & 0.0000 & 7.7953 & 0.0002 & 1.3484 & - & - \\
Weibull & 0.1436 & 0.6532 & 0.7467 & 0.5196 & 2.3089 & - & 26.0230 \\
\hline \hline
\end{tabular}

\section{Acknowledgements}

This paper is benefited from Dr. Chookait Pudprommarat from Faculty of Science and Technology at Suansunandha Rajabhat University. His comments and suggestions are gratefully acknowledged. We are grateful to the Commission on Faculty of Science Kasetsart University Postgraduate Studentship (ScKUPGS), Thailand, for supporting the Scholarships.

\section{References}

[1] W. Weibull, A statistical distribution function of wide applicability, $A p$ plied Mechanics, 18, No. 3 (1951), 293-297.

[2] J.F. Lawless, Statistical Models and Methods for Lifetime Data, 2nd ed, Weiley, Canada (2003), doi: 10.1002/9781118033005.

[3] D.N.P. Murthy, M. Xie, R. Jiang, Weibull Models, John Wiley and Sons, Canada (2004), doi: 10.1002/047147326X.

[4] A. Keller, A. Kamath, Alternative reliability models for mechanical systems, 3rd International conference on Reliability and Maintainability, Toulouse, France (1982).

[5] P. Erto, Properties and identification of the inverse Weibull lifetime model (in italian), Statistica Applicata, 1, No. 2 (1989), 117-128.

[6] M.S. Khan, G.R. Pasha, A.H. Pasha, Theoretical analysis of inverse Weibull distribution, WSEAS Transactions on Mathematics, 7, No. 2 (2008), 30-38. 


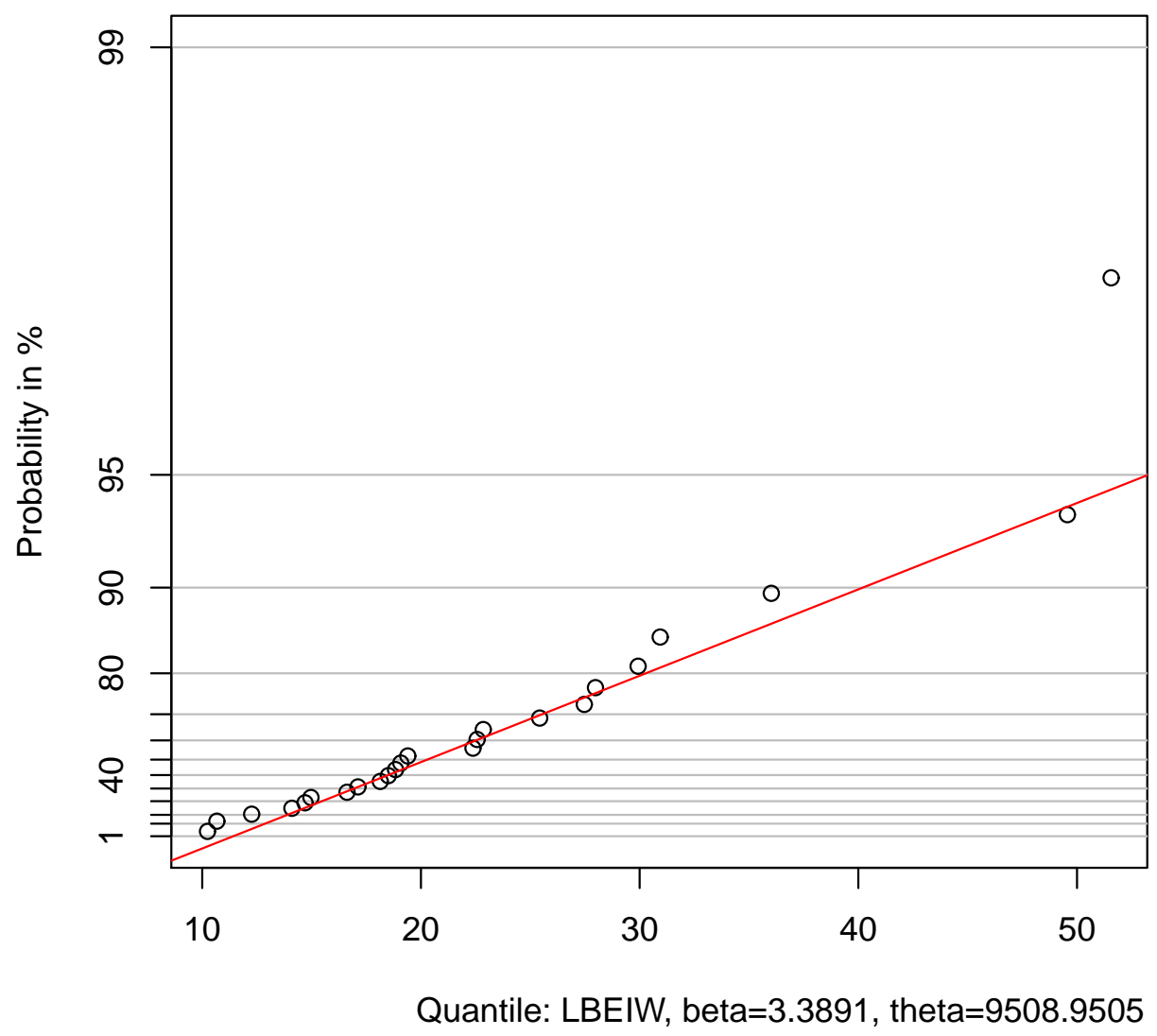

Figure 4: The probability plot for the model based on the length-biased exponentiated inverted Weibull distribution applied to the distance between cracks in a pipe dataset.

[7] G. Mudholkar, D. Srivastava, Exponentiated Weibull family for analyzing bathtub failure rate data, IEEE Transactions on Reliability, 42, No. 2 (1993), 299-302, doi: 10.1109/24.229504.

[8] G.S. Mudholkar, D.K. Srivastava, G.D. Kollia, A generalization of the Weibull distribution with application to the analysis of survival data, American Statistical Association, 91, No. 436 (1996), 1575-1583, doi: 
10.1080/01621459.1996.10476725.

[9] R. Gupta and D. Kundu, Generalized exponential distributions, Australian and New Zealand Journal of Statistics, 41, No. 2 (1999), 173-188, doi: 10.1111/1467-842X.00072.

[10] A. Flaih, H. Elsalloukh, E. Mendi, M. Milanova, The exponentiated inverted Weibull distribution, Applied Mathematics and Information Sciences, 6, No. 2 (2012), 167-171.

[11] D. Cox, Renewal Theory, Barnes and Noble, New York (1962).

[12] R.C. Gupta, H. Akman, On the reliability studies of a weighted inverse Gaussian model, Statistical Planning and Inference, 48, No. 1 (1995), 6983, doi: 10.1016/0378-3758(94)00148-O.

[13] G.P. Patil, C.R. Rao, Weighted distributions and size-biased sampling with applications to wildlife populations and human families, Biometrics, 34, No. 2 (1978), 179-189.

[14] K.K. Das, T.D. Roy, On some length-biased weighted Weibull distribution, Advances in Applied Science Research, 2, No. 5 (2011), 465-475.

[15] K.K. Das, T.D. Roy, Applicability of length biased weighted generalized Rayleigh distribution, Advances in Applied Science Research, 2, No. 4 (2011), 320-327.

[16] K. Mir, A. Ahmed, J. Reshi, Structural properties of length biased beta distribution of first kind, American Jounal of Engineering Research, 2, No. 2 (2013), 1-6.

[17] M. Pandya, S. Pandya, P. Andharia, Bayes estimation of Weibull length biased distribution, Asian Journal of Current Engineering and Maths, 2, No. 1 (2013), 44-49.

[18] Z.W. Birnbaum, F.H. Tingey, One-sided confidence contours for probability distribution functions, Annals of Mathematical Statistics, 22, No. 4 (1951), 592-596.

[19] G. Chen, N. Balakrishnan, A general purpose approximate goodness-of-fit test, Quality Technology, 27, No. 2 (1995), 154-161. 